Avec une pècherie bien organisée, pècher n'est plus une épouvantable corvée qu'on recule d'année en année sans pouvoir s'y décider. C'est devenu un travail normal permettant la cueillette d'un intéressant bénéfice.

Mais que dira-t-on maintenant du nouveau système que vient d'installer notre co!lègue M. Alland, de Nenigoulte (Deux-Sèvres) à son bel élang des Chatelliers? Profitant du fail que le ruisseau, au sortir de l'ćlang présentait un: pente notable, il a adapté au-dessus du fond de la pêcherie un caillebotis horizontal en bois dûment raboté et lissé et de dimensions telles que le poisson qui arrive par le canal de bonde s'y trouve lavé et mis à sec et qu'il n'y a plus qu'à le ramasser comme l'indique la photographie. (Figr. 16).

L'ensemble est installé sous une tente qui, pour le travail de nuit est éclairée électriquement.

Nous voici loin de la laboricuse pêche à la senne dans la boue d'un étang vidé aux $9 / 10^{\text {es }}$ et plus près, ne vous semble-t-il pas de la fameuse usine de Chicago où le porc entier entre dans une machine qui le restitue à l'autre bout converti en jambon, saucisses ef boudins tout cuits?

A quand la pèche en gants blancs?

\title{
AU SUJET DE LA LUNE ET DE SON INFLUENGE
}

\author{
Par Michel LHÉRITIER
}

Pisciculteur is Ambazac (Haute-Vienne).

Au prinlemps dernier, j’ai lu dans la Causeric scientifique d'un granł journal parisien, un article fort intéressant dans lequel l'auteur, savant connu, nic totalement l'influence de la Reine des Nuits sur les êtres et les choses de notre planète et qualifie d'ignorants ceux qui croient en cette influence.

Bien que je sois de ceux-ci, et que l'épithète ne me blesse en aucune manière, je me proposai de soumellre à l'attention de ce personnage quelques réflexions suggérées par des faits vécus et contrôlables, susceptibles, sinon de lui faire réviser son jugement, tout au moins de jeter quelque trouble dans son esprit et de le rendre un peu plus tolérant pour ses contradicteurs.

Mais, n'ayant point eu alors le Ioisir d'écrire, j'cvais complètement oublié ce projet de réplique, quand un récent article de la Revue scientifique du Limousin (I), remettant le sujet en question, me donna l'idée de l'exécuter.

(1) No 384 , Juillet-Aoùt 1934 . 
J'y suis, du reste, encouragé par la lecture d'un article du très savant abbé Moneux intitulé : "Comment la lune agit-elle sur nous ? " reproduit dans la susdite Revue, et dont je crois donner quelques extraits.

"Tout le Moyen Age a cru à certaines influences lunaires sur les orga" nismes vivants. Au nom d'une science un peu simpliste parce que trop " ignorante, les "Scientistes " des deux derniers siècles décrétèrent qu'il " n'en n'était rien et que cette action prétendue de la lune n'était qu'une " légende née au hasard dans l'esprit d'un peuple crédule.

"A vrai dire, on trouve chez les savants de tous les tcmps un défaut " capital dont ils ne peuvent se débarrasser : les théories de leur époque " influent tellement sur leurs constatations qu'ils en arrivent à déformer " les faits et même à ne plus les voir parce qu'ils sont impuissants à les " expliquer.

"N'ai-je pas vu des astronomes notoires nier, il y a vingt-cinq ans, l'influence des taches solaires sur notre climatologie, sous prétexte qu'une relation de cause à effet eût été inexplicable ? Aujourd'hui que nous avons mis en évidence une émission constante de corpuseule électrisés, lancés par le soleil et, en particulier, par ses faches et ses protubérances, c'est a qui se glorifiera d'apporter des faits pour corroborer mes théories.

"En est-il de même pour la lune ? Pas tout à fait. Aussi je me rappelle le beau tapage que suscita la publication de mon ouvrage : "Un jour dans la lune ". Josais, pour la premiere fois depuis bien longlemps, sinon réhabiliter toutes les anciennes croyances sur l'influence de la lune, montrer du moins qu'elles n étaient pas aussi dénuées de fondement que l'avait admis une science infatuée d'elle'mème".

Je ne me serais pas permis de parter de !a sorte, mais javoue avoir pensé et penser encore ce que dit si éloquemment l'éminent directeur de l'Observatoire de Bourges.

Je ne veux pas revenir sur le cas des " lunatiques " dont parle cet auteur, ni des phénomènes dont nos compagnes constatent périodiquement les manifestations ; je veux simplement signaler à mes lecteurs un ensemble de constatations faites durant 35 années de pratique de la pisciculture et qui pouront peut-ètre rendre service à nos collìrues exploitants d'étangs, tout en montrant, d'une manière évidente, l'influence de la lune sur les poissons et probablement sur tous les êtres de la terre.

Si, pendant la plus grande partie de l'année, la nature garde jalousement se's secrets sur la vio et les mours des animaux sauvages, il vient cepentant tous les ans une période, plus ou moins longue, où l'homme peut facilement suinstruire sur les habitudes des bêtes contre lesquelles il a à se défendre comme de celles dont il se nourrit ou s'amuse, c'est quand la neige recouvre la terre. Sur la page blanche de cet immense livre, les malheureuses créatures inscrivent elles-mêmes leur nom, leur adresse et révèlent les ruses qu'elles emploient journellement pour échapper à leurs innombrables ennemis. 
Or, tous ceux qui, durant l'hiver, ont suivi les rives des cours d'ear et des étangs recowvertes de neige ont pu constater qu'en lune nouvelle ( ${ }^{\text {er }}$ quartier) les traces de pas marquées par la Loutre se dirigent de l'aval vers l'amont, tandis que celles reconnues durant la lune vieille (dernier quarticr) se dirigent au contraire de l'amont vers l'aval, ce dont ne manquent pas de s'inspirer les piégreurs habiles; dans le premier cas, ils tendent leurs engins en aval de la pierre blanche ou du petit monticule sur lequel la Loutre va déposer ses épreintes, alors que, dans le second cas, la tendue se fait du côté amont. Ces déplacements périodiques sont tellement habituels à la Loutre que, sauf dans re cas d'un animal cantonné ou accidentellement dérangé, il est absolument inutile de tendre les pièges en dehors des huit jours finissant et précédant la période lunaire.

Or, l'chervations séculaires transmises de génération en génération, ii résulte que ces mocurs si singulières de la Loulre ne sont que des migrations alimentaircs découlant d'un phénomène identique qui se manifeste chez les habitants de l'onde.

Dans notre Limousin, dont la plupart des nombreux étangs sont alimentés par des cours d'eau leur donnant une grande analogie avec les rivières, tous les aquicultcurs avertis reconnaissent l'exactitude de ce vieux dicton : " en lune nouvelle, le poisson morte; en lune vieille, le poisson descend; " aussi les exploitants d'étangs s'appliquent-ils à exécuter Ieurs pèches entre le plein de la lune et la fin de son dernier quartier. Je puis fournir de nombreuses preuves des avantages de ce système, tant au point de vue de la capture du poisson que de cclle de notre vieille ennemie : la Loutre ; je ne saurais done trop engager nos collègues pisciculteurs el aquiculteurs à l'expérimenter, puis plus tard à communiquer à notre Bulletin le résultat de leurs observations personnelles.

Ainsi, par exemple, jai cu, durant plusicurs années, la direction totale de deux grandes exploitations carpicoles de la Ramade (Creuse), appartenasit à M. le docteur 1. Samivox, et de Tyx (Puy-de-Dôme), propriété de M. Emile Chapai, dans lesquelles j’avais instauré la pratique des pêches annuclles. Les étangs de la Ramade (80 hectares), ct celui de Tyx (75 hectares) sont tous deux de forme très allongéc et alimentés chacun par deux forts ruisseaux actionnant des moulins, et on y capture le poisson en faisant descendre en aval de la chaussée lout le peuple de l'étang; j'avais complètement supprimé la coutume du " passage de la torche " jusqu'alors réputé indispensable au refoulement du poisson dans les appareils de pèche.

Celle opéralion de " torchage ", encore en honneur dans beaucoup d'endroits, consiste à établir, avec de légers branchages, une sorte de long fagot d'environ $o^{\text {in }}$ io de diamère et d'une longueur correspondant à la largeur rlu cours d'ecuu coulant dans le fond de l'étang, puis à le poser, comme un barrage, en travers de ce ruisseau, au-dessus de la nappe dans laquelle sont. rassemblés les poissons par suite de la vidange presque complète de l'étang, 
'c'est-à-dire au moment où on le qualifie "en pêche ". Alors, la bonde étant ouverte complètement, des hommes viruoureux, vêtus pour la circonstance et copieusement abreuvés de vin chaud, prennent, en nombre suffisant, place derrière la torche et la poussent devant eux, refoulant à la fois vase et poissons vers la bonde qui les avale.

Suivant les circonstances, l'opération sc répèto jusqu'à ce qu'il ne reste plus de poissons de valcur dans l'étang; on a soin, pour ce faire, de fermer l'étang après chaque " coup de torche " afin de faire remonter un peu l'eau dans laquelle pourront se rassembler les poissons laissés en traîne.

Il est évident que, pour le public assistant à la pêche, le " passage de lat torche " est un spectacle très pittoresque et amusant à cause des incidents comiques qu'il provoque ; mais pour le Poisson qu'il malmène fort ct asphyxic plus ou moins complètement, comme aussi pour l'exploitant, c'est une opération dangereuse parce qu'elle expose les hommes à prendre mal, qu'elle meurtrit de pauvres hêtes sans défense, ce qui rend leur conservation aléatoire, et qu'elle augmente dans une large mesure les frais habituels de pêche.

Un autre gros inconvénient du " torchage " c'est d'accumuler, dans un rayon proche de la bonde, une quantité considérable de vase qui, à la pêche suivante, nuira à la vidange, empêchcra la descente du poisson et obligera à recourir encore à ce procédé barbare.

Or, dans' les deux exploitations précitées, les pêches par vidange ayant toujours lieu à la fin de la lune d'Octobre, ou de celle de Novembre au plus tard. les poissons descendaient toujours et complètement dans les fosses sans qu'on ait recours à un autre artifice qu'une vidange régulière exécutée entre le plein et le revif de la lune; c'est-à-dire en lune vieille.

Quand je prérise ri-dessus : lune d'Octobre ou de Novembre, je ne veux pas dire que l'influence de ce satellite ne se manifeste pas dans les autres mois, mais simplement qu'à l'altitude où sont situés ces étangs les intempéries imposent de pêcher avant l'hiver.

En revanche, dans la même région, malgré des passages réitérés de torche, j'ai dû. une fois, laisser la moitié des alevins de l'étang de Franouille, dépendant de l'exploitation de la Ramade, et, une autre fois, capturer dans l'intérieur même de l'étang de Gasserot, dépendant de l'exploitation de Tyx, avec des engins appropriés, des " nourrains" de 2 étés. Mes aides de cette équipée peuvent en témoigner.

Quant à la Loutre, voici, parmi nombre d'autres, deux observations faites en milieux différents :

A l'exploitation carpicole de la Ramade, il existe, entre la chaussée et l'arche où se prennent les $A$ guilles, un tronçon de rivière d'une centaine de mètres. dont une partic sur la rive droitc est plantée d'une rangée de bouleaux. Entre ces arbres ct la rivièrc, profonde de 60 centimètres environ, existe une petite bordure constituée par un mur en grosses pierres 
recouvertes de gazon, et servant de retraite aux Anguilles sorties de l'étang.

Or, ayant observé que la Loutre affectionnait particulièrement passer sur cette banquette, j'y ai pratiqué, à quelques décamètres l'une de l'autre, deux petites excavations permettant de tendre facilement, et sous dix centimètres d'eau, un piège à palette muni d'une chaîne assez longue pour permettre à la bête prise de se noyer rapidement ; eh bien ! la consultation des notes quotidiennes, dont j'ai imposé la tenue aux gardes, démontre que les quatre cinquièmes des Loutres cąpturées en cet endroit l'ont été dans la huitaine de jours finissant ou commençant la lune (quatre avant et quatre après).

Mon établissement de pisciculture installé, depuis rgo6, dans un étranglement de la vallée du Beuvret, affluent du Taurion, est limité au sud par une route passant sur un pont sous lequel circule le ruisseau longeant mes hassins côté ouest et, au nord, par l'étang du Petit-Jonas, dominé luimême par les monts d'Ambazac et les étangs de Grammont.

Or, pendant la création de mon établissement, un vieux braconnier du pays, me voyant prendre des dispositions de défense contre la Loutre, me rappela : "En lune nouvelle, les Ioutres du Taurion montent dans les étangs de Grammont et redescendent en lune vieille au Taurion ". J'ai donc pris mes mesures en conséquence et, sur une quinzaine de captures faites depuis cette époque, une douzaine ont eu lieu dans la période précitée ( $\mathrm{r}$ ).

Quant à la raison qui pousse les poissons à pérégriner ainsi, j'avoue humblement que je l'ignore ; mais, il y a là un fait incontestable qui me paraît donner aux savants quelques idées qu'il serait sans doute intéressant de connaître.

Dans le règne végétal, j'ai fait aussi quelques constatations que je crois dignnes d'intérêt : telle, par exemple, la forme des branches d'un jeune arbre taillé en lune nouvelle ; dans re cas, les branches qui repoussent, au lieu de s'élancer obliquement vers le ciel, commencent d'abord par s'incurver vers la terre pour se redresser ensuite verticalement, comme les branches d'un candélabre.

Dans les jours, hélas ! lointains, de mon enfance, j'ai souvent entendu dire aux vieux fagotiers exploitant les taillis de chêne environnant Aubusson que les liens, constitués par de jeunes pousses, devaient impérieusement être coupés en vieille lune, faute de quoi ils n'étaient pas utilisables parce que : cassants. Il est facile à chacun de constater que le bois le plus souple, l'osier, lui-même, ne se travaille pas également en lune nouvelle et en lune vieille.

(1) Ces déplacements périodiques de la Loutre font croire à l'abondance de cet animal qui, heureusement pour les poissons et leurs éleveurs, se raréfie de plus en plus et finirait même par disparaître complètement si on la traquait avec un peu de méthode. 
Pour finir, je crois devoir citer un autre exemple de l'influence de la lune sur les végétaux comportant une explication presque scientifique de se phénomène.

Si les hommes d'une cinquantaine d'années consultent leurs souvenirs, ils peuvent se rappeler avoir vu construire des maisons dont les possesseurs actuels sont déjà dans l'obligation de réparer les planchers et les charpentes dévorés par les vers ; or, tout autour d'cux, ils peuvent voir d'autres maisons vieilles de plusieurs siècles dont les charpentes sont encore intactes et dont les réparations dẹ planchers, quand il y en a eu, n'avaient pour cause que l'usure.

Voici comment j'explique la chose : quand, durant une promenade en ampagne et à travers les taillis, on casse, puis porte à la bouche une jeune pousse d'arbre ou arbusle quelcongue, on lui trouve quelque fois une saveur sucrée assez agréable, landis que d'autre fois elle est complétement insipide ; or, dans le premier cas, qui ne se produit qu'en période de lune nouvelle, c'est la présence de la sève circulant sous l'écorce qui cause la saveur agréable constatée, par suite de sa richesse en saccharose; plus tard, au contraire, en période de lune vieille, rette saccharose, nourrissant le bois, s'étant transformée en cellulose, on comprend alors que la jeune pousse n'ait plus de goût.

Or, nos pères, plus sages ou moins pressés que nous du désir de jouir, ayant l'habitude d'abaltre toujours les bois d'cuvre en lune vieille, alors que la sève transformée en cellulose n'intéressait plus les insectes qui en vivent, leurs planchers et leurs charpentes bravaient impunément les siècles, tandis que, de nos jours, les bois, ahattus le plus souvent sans aucun souci de l'époque, sont souvent aussi riches en sève dont la saccharose recherchée par les Insectes provoque lcurs attaques qui en causent la ruine.

De cela, il semble résulter que : toute l'annéc, un flot de sève plus ou moins considérable, suivant les saisons, circule dans les végétaux sous l'influence de la lune, laquelle influence, je le rappelle, se manifeste dans se règne animal, par les menstrues des femmes, le rut des animaux, ainsi que les périodes de gestation et d'incubation qui sont déterminées par la durée des phases lunaires, leurs multiples ou sous-multiples.

Ne suis-je donc pas fondê de croire en l'influence de la lune sur les êtres vivants? 